\title{
Desafíos para el profesorado en la sociedad del conocimiento
}

\author{
Challenges for teachers in the knowledge society \\ Liliana Pedraja Rejas ${ }^{1}$ \\ Recibido 15 de marzo de 2011, aceptado 26 de abril de 2012 \\ Received: March 15, $2011 \quad$ Accepted: April 26, 2012
}

\begin{abstract}
RESUMEN
El presente trabajo tiene como objetivo identificar y describir las principales características de la sociedad del conocimiento que deben ser consideradas por el profesorado en el ejercicio de su actividad de enseñanza. A partir de estos rasgos distintivos de la sociedad del conocimiento se generan un conjunto de desafíos y roles que los profesores deben asumir para ser efectivos en su labor formadora en la sociedad actual. Finalmente, se discuten las principales tensiones que emergen en el profesorado al asumir los imperativos estratégicos que demanda la sociedad del conocimiento.
\end{abstract}

Palabras clave: Docencia, rol del profesorado, sociedad del conocimiento, imperativos estratégicos, educación.

\section{ABSTRACT}

The present work aims to identify and describe the main characteristics of the knowledge society, that must be considered by the teachers in carrying out their teaching. Based on these distinctive features of the knowledge, generated a set of challenges and roles that teachers should take to be effective in keeping the formation in today's society. Finally, the main tensions that emerged in the faculty in order to assume the strategic imperatives that demand knowledge society were discussed.

Keywords: Teaching, teacher role, knowledge society, role of teachers, education.

\section{INTRODUCCIÓN}

Antiguamente se pensaba que el conocimiento debía ser protegido, así se recuerdan sociedades que pretendían obtener una ventaja comparativa mediante el manejo de la información.

Hoy, en cambio, la información está disponible no sólo para pequeños grupos de personas como en el pasado, sino que se encuentra al alcance de centenares de millones de personas a través de Internet. Al respecto, Rodríguez Ponce [31] sostiene que existen cientos de millones de páginas electrónicas en la red; se envían alrededor de 60 mil millones de correos electrónicos diariamente y funcionan más de 73 millones de blogs. Asimismo, en la actualidad existen más de 100 mil publicaciones científicas asociadas a redes formales, a las que se puede acceder en diferentes formatos; pero adicionalmente en la red, más allá de las publicaciones tradicionales, es posible acceder a más de 300 mil páginas que corresponden a trabajos científicos.

Los profesores, en tanto, se ven insertos en un contexto de cambios permanentes, sistemáticos e ininterrumpidos. No es menor el hecho de que la mayoría de los profesores nacieron y se educaron en la era del progreso industrial y hoy se encuentran viviendo un nuevo tiempo, llamado sociedad del conocimiento, en el cual los paradigmas tecnológicos, sociales y económicos han cambiado significativamente.

1 Escuela Universitaria de Ingeniería Industrial, Informática y de Sistemas. Universidad de Tarapacá. Arica, Chile.

E-mail: lpedraja@uta.cl 
En este trabajo se pretende identificar: ¿cuáles son los aspectos que caracterizan a la sociedad del conocimiento?; ¿cuáles son los principales roles que debe asumir un profesor para tender hacia la efectividad en la llamada sociedad del conocimiento?; ¿qué tensiones se producen al incorporar dichos roles? La respuesta a estas interrogantes puede contribuir a generar imperativos estratégicos para delinear el rol del profesor en la sociedad actual, en la perspectiva de formar escolares hábiles y capaces para desempeñarse en la sociedad del conocimiento. Para este efecto, se desarrollan algunos planteamientos fundamentales que permiten comprender qué es la sociedad del conocimiento y cómo esta sociedad impacta y genera desafíos para la formación de capital humano en los países. Seguidamente, se describen las principales características de la sociedad del conocimiento que impactan sobre el rol del profesorado. Consecuentemente, se definen estos roles del profesorado en la sociedad del conocimiento y se analizan las tensiones que emergen en la ejecución de los roles y desafíos de los profesores.

\section{LA SOCIEDAD DEL CONOCIMIENTO}

Drucker [11] indicó que la sociedad del conocimiento será cada vez más competitiva, dado que el conocimiento es ahora universalmente accesible. En la lógica de Drucker, no existirán países pobres sino sólo países incapaces de crear, adquirir y/o aplicar el conocimiento. Así, las sociedades requieren de personas con educación continua; capacidad de adaptación; buenas prácticas de trabajo, y nuevos modelos de interacción al interior de las organizaciones. Por tanto, una primera característica de la sociedad del conocimiento es el alto grado de competencia que existe entre las naciones, las organizaciones y las personas. Esta competencia se ve acentuada por la globalización de la economía mundial y por la apertura de los mercados financieros y de los mercados de producción y comercialización de productos y/o servicios.

El nivel de conocimiento requerido para funcionar con eficacia social es muy elevado si se compara con los niveles necesarios en la segunda mitad del siglo anterior. Consecuentemente, se requiere de una escolarización también larga. Por ejemplo, hasta un 70\% de jóvenes acuden en Japón o Estados Unidos a la educación terciaria, con lo cual la educación superior en la sociedad del conocimiento es equivalente a lo que la enseñanza media era en la sociedad industrial, siendo el doctorado el equivalente a lo que antes era la formación universitaria [21].

El sentido más profundo del término sociedad del conocimiento, es que si antes la cultura era la ciencia, hoy la ciencia es la cultura dominante que incide en el quehacer integral de toda la humanidad. Así, una segunda característica de esta sociedad del conocimiento es que para ser un sujeto eficaz y hábil socialmente hablando se requiere una creciente formación de tipo formal. Las personas incapaces de lograr estos niveles de formación se quedarán al margen de una economía donde el éxito se mide por la productividad, la capacidad de innovación y la creación de valor estratégico.

La nueva sociedad conduce a una nueva economía del conocimiento, la que se caracteriza porque el conocimiento es el elemento fundamental para generar valor y riqueza en las organizaciones y en la propia sociedad. El conocimiento es más que información, ya que el conocimiento incorpora formas y métodos para resolver problemas en los distintos ámbitos del quehacer de las organizaciones. La capacidad de creación de valor a partir del conocimiento es esencialmente intangible y ésta es la fuente de la ventaja competitiva en la sociedad actual. Por tanto, el conocimiento en sus más amplias vertientes es la base de la economía actual.

Una tercera característica de esta sociedad del conocimiento es que la fuente de la ventaja competitiva radica en las personas. El capital humano avanzado es la base de la ventaja competitiva en la medida que sea capaz de crear, compartir y aplicar conocimiento [20].

Por su parte, la educación juega un rol fundamental en la actual sociedad del conocimiento. En efecto, considérese que a nivel global un año más de escolaridad tiene un impacto sobre la productividad individual que bordea entre el 6\% y el 15\% [5, 23]; pero ese mismo año adicional de escolaridad implica un incremento del potencial del producto interno bruto en proporciones que, en el largo plazo, resultan altamente significativas $[10,15]$. Complementando lo anterior, se sabe que la educación terciaria constituye una fuente de movilidad social con una rentabilidad privada muy atractiva para las personas de los países emergentes [12], incluso en localidades 
extremas [8]; entonces, se puede aseverar que para las naciones, las organizaciones empresariales y sin fines de lucro y para las personas el conocimiento es una fuente de creación de valor y de generación de ventaja competitiva.

Sin embargo, si bien la evidencia empírica en Chile muestra que siete de cada diez estudiantes que están en educación superior son primera generación en obtener tal logro académico, no es menos cierto que cinco de cada diez estudiantes que llegan a la educación superior no finalizan sus estudios con éxito. No basta con acceder a la educación superior, se requieren los conocimientos suficientes y las competencias necesarias para conseguir éxito en este nivel de formación [31].

Una cuarta característica de la sociedad del conocimiento, por tanto, es que la formación de capital humano avanzado requiere de ciertas destrezas, conocimientos y habilidades que deben lograrse en la enseñanza media y básica.

Más aún, el conocimiento ha sido siempre un factor de producción; no obstante, en la actualidad la capacidad de administrar, almacenar y transmitir grandes cantidades de información a bajo precio es un elemento central de los procesos organizativos y de la sociedad. En este contexto, el conocimiento otorga poder a quienes lo poseen, y proporciona capacidad de acción y decisión. Consecuentemente, hoy la fuente de la ventaja competitiva reside en la capacidad para adquirir, transmitir y aplicar el conocimiento.

Una quinta característica de la sociedad del conocimiento es que las tecnologías de información y comunicación son un medio esencial para el accionar diario y efectivo de las personas tanto en el mundo del trabajo como en el campo de las relaciones sociales. Además, estas tecnologías son esencialmente digitales. En este contexto, la sociedad del conocimiento se combina con la globalización. La globalización es un proceso que comprende múltiples dimensiones, tales como los aspectos económicos, sociales, políticos y culturales. Sin embargo, su rasgo más determinante es la interdependencia de los mercados, favorecida por las nuevas tecnologías de información y comunicación y la apertura de esos mercados. La simultaneidad e instantaneidad de los acontecimientos es otro de los rasgos que caracterizan la globalización. Globalización significa que las personas se relacionan directamente en una esfera social, donde las fronteras geográficas pierden relevancia, y estas fronteras se ven superadas por las nuevas tecnologías de información. Las relaciones globales ocurren, a veces, sin regulación por parte de los Estados, generándose entonces una dimensión más amplia que el territorio físico para comprender lo que es ser ciudadano en los tiempos actuales [6]. En este contexto, las personas participan en redes sociales a través de Internet. La sociedad en red facilita el teletrabajo, la autoinstrucción, el conocer otros mundos de oportunidades y otras realidades desde el mismo hogar, permitiendo que la información esté al alcance de la mano de manera fácil, segura, y cada vez más confiable [16].

Por ello, una sexta característica de esta sociedad del conocimiento es la apertura a una sociedad global y de trabajo en redes que superan la esfera geográfica. Ciertamente, estas características se exponen sin ánimo exhaustivo, sino que más bien como algunos de los aspectos relevantes que configuran los desafíos estratégicos que deben asumir los profesores para una labor docente eficaz.

A partir de las características antes enunciadas es posible establecer algunos requerimientos para los estudiantes en términos de capacidades para:

- Enfrentar un mundo con altos niveles de competencia a nivel de países, organizaciones y personas.

- Lograr niveles elevados de educación, y capacidad para adquirir formación continua o permanente.

- Aprender y contar con conocimientos y competencias para desempeñarse exitosamente en procesos de formación superior y educación continua.

- Manejar funcionalmente las nuevas tecnologías de información y comunicación.

- Trabajar en redes, más allá de las esferas geográficas predefinidas.

\section{LOS ROLES DEL DOCENTE EN LA SOCIEDAD DEL CONOCIMIENTO}

A partir de los desafíos estratégicos que deben asumir los estudiantes en la sociedad del conocimiento, emergen los roles para el profesorado. 


\section{El profesor como facilitador}

Para preparar a los jóvenes a enfrentar una sociedad con una competencia creciente, tanto a nivel de naciones como de organizaciones y personas, se requiere que los profesores asuman un rol de facilitador.

Efectivamente, el profesor debe preparar a los estudiantes para abordar un mundo de creatividad y flexibilidad y, consecuentemente, el profesor debe protegerlos contra las amenazas de la competencia que surge en la sociedad actual [17].

De modo similar, el profesor debe estimular a los estudiantes a reconocer la realidad competitiva de la sociedad, para que los alumnos entiendan la importancia de alcanzar cada vez niveles de educación más altos. Al mismo tiempo, debe apoyar y facilitar su aprendizaje y debe prepararles para asumir con éxito la educación superior. El aprendizaje es un proceso complejo en el que interactúan un conjunto amplio de variables, el profesor debe ser quien asista o apoye a los estudiantes en su aprendizaje. Los aspectos externos e internos que subyacen en el proceso de aprendizaje deben ser comprendidos y asumidos por el profesor, pues sólo de este modo se podrá llevar a cabo el rol de facilitador [33].

En términos más concretos, Oliver sostiene que: "El profesor debe actuar más como animador e investigador del entorno y como motivador y puente entre el medio ambiente de la población escolarizada, el mundo científico y cultural que como transmisor y reproductor a priori de los contenidos que de este mundo dimanan, ello justifica algunas exigencias al profesorado, de una parte, y al currículum de formación, de otra" [28].

Por tanto, un profesor facilitador debe colaborar con los estudiantes en la construcción de sus conocimientos y capacidades, pero no sólo como un monitor y evaluador de los resultados obtenidos. Este rol demanda un compromiso con el proceso de enseñanza, y con los resultados del aprendizaje. Los profesores no sólo deben entregar conocimiento, sino que deben desarrollar en los estudiantes hábitos, disciplina, motivación y, además, crear un ambiente de colaboración y facilitación de su aprendizaje [17]. En efecto, bajo esta línea de razonamiento, Esteve [13] argumenta que el profesor debe reconvertir su trabajo de clase, hacia la facilitación del aprendizaje y la orientación del trabajo del alumno, puesto que este último llega al aula con una predisposición a discutir y confrontar los conocimientos recibidos con la información que recogió previamente desde la televisión o Internet.

No obstante lo anterior, Sarason [32] establece que los programas de formación, dejando de lado su retórica, preparan en forma incorrecta a los profesores en relación a su rol, la disyunción y las formas de enfrentarlo [2].

\section{El profesor como gestor del conocimiento}

Para preparar a los jóvenes, en la perspectiva de lograr éxito en su educación superior, así como para dominar funcionalmente las tecnologías de información y comunicaciones, y trabajar eficazmente en un contexto de redes globales, se requiere que el profesor sea un gestor de conocimiento.

En efecto, el profesor en la sociedad del conocimiento debe ser capaz de dominar los conocimientos referidos a su disciplina; pero además debe ser capaz de gestionar los medios que tiene a su alcance de cara a tornar dinámico el aprendizaje de sus estudiantes y prepararlos para enfrentar un mundo globalizado, en el cual las esferas geográficas han sido superadas por redes globales que trabajan en el ambiente de Internet.

Un profesor gestor de conocimiento debe ser capaz de dominar su disciplina y especialidad, pero además, debe ser capaz de compartir su conocimiento con los estudiantes, recibir el conocimiento de los estudiantes, y buscar en conjunto las aplicaciones que la evidencia empírica muestra para dicho conocimiento.

En este sentido, Villanueva [36] plantea que el rol del profesor se debe orientar a que los estudiantes tiendan a "aprender a aprender para emprender y ser", en vez de sólo enseñar conceptos que tendrán vigencias limitadas y que estarán siempre accesibles para quienes los necesiten.

Marcelo [25] sostiene que se requiere replantear las funciones tradicionalmente asignadas a las escuelas $\mathrm{y}$, en particular, a los profesores, puesto que las fuentes de información aportadas por las nuevas tecnologías de la información y comunicación 
permiten acceder en tiempo real a un conjunto de conocimientos antes no disponibles.

A este respecto, Uibu y Kikas [34] indican que la introducción de tecnología de información y comunicación en la educación ha tenido un impacto en la percepción de la función del profesorado; sin embargo, y a través de un estudio cualitativo, obtienen información que da luces acerca de cambios en la forma de llevar a cabo las clases, mas no de cambios en cuanto al fondo, para el caso de profesores de educación primaria. Fainholc [14] propone que el rol de profesor debe centrarse en la construcción de conocimiento como una habilidad adicional, pero vinculado más a la transformación de la información que a la repetición y la distribución del mismo. Ciertamente, tal y como lo plantean Alavi y Leidner [1], la gestión del conocimiento tiene como objetivo fundamental soportar la creación, la transferencia y la aplicación del conocimiento en las organizaciones.

En el campo de la educación, dicha gestión se refiere a crear, divulgar y utilizar el conocimiento, pero con el propósito central de lograr el mejoramiento de la enseñanza y el aprendizaje de los estudiantes. Para llevar el rol de gestor del conocimiento a la acción, resulta vital poder educar a los estudiantes promoviendo el desarrollo de sus capacidades bajo una mirada de coconstrucción. El enfoque constructivista ha sido útil para aquello. La experimentación y la autonomía forman parte de gestionar el conocimiento en las aulas. De este modo, Marabotto [24] indica que el docente interesado en mejorar su tarea debe ser capaz de evaluar estrategias cognitivas que resulten más eficaces para la difusión de conocimiento entre sus estudiantes. Un profesor gestor de conocimiento promoverá en sus estudiantes la adquisición de capacidades individuales para ser los propios creadores y gestores de sus conocimientos, tanto en la disciplina específica como en los diferentes frentes en que los estudiantes participan en la sociedad de redes y ampliamente globalizada.

\section{El profesor como líder transformacional}

Los roles de facilitador y gestor del conocimiento confluyen en un nuevo rol de liderazgo, esencialmente, transformacional del profesor. En efecto, el liderazgo transformacional engloba a un estilo que desarrolla un tipo de líder capaz de ayudar a tomar conciencia de los demás de sus posibilidades y capacidades reales bajo un enfoque participativo. El líder transformacional logrará que sus estudiantes sean capaces de comprender el ambiente competitivo en el que viven. A la vez, los estimulará para adquirir una formación elevada que les permita interactuar con eficacia social. Asimismo, el profesor como líder transformacional dotará a los estudiantes de los conocimientos y capacidades para enfrentar con éxito la educación superior, y su actuación en redes globales, con un dominio funcional de las tecnologías de información y comunicaciones.

Carisma, visión o capacidad de formular una misión, consideración individual, es decir, atención a las diferencias personales y a las necesidades diversas, estimulación intelectual, y capacidad para motivar, potenciar las necesidades y proporcionar un apoyo intelectual y emocional, son algunos de los atributos que debe cumplir el profesor en este rol [4]. En el contexto de la sala de clases, evidentemente el profesor asume un rol de liderazgo; sin embargo, la propuesta de ejercer un liderazgo de tipo transformacional implica un rol más específico, vale decir, el ejercer un liderazgo capaz de realizar cambios basados en las capacidades que poseen los estudiantes. Recientemente se ha probado la importancia del liderazgo transformacional en las escuelas chilenas [29]. En forma equivalente, Van den Berg [35] descubre que el liderazgo transformacional es el factor clave para llevar a cabo procesos de innovación en las escuelas.

Hargreaves y Fink [18-19] proponen la eficacia del líder sustentable que es aquel que genera y mantiene un aprendizaje sostenible; asegura el éxito en el tiempo; apoya el liderazgo de otros; dirige su atención a la justicia social; desarrolla, más que utiliza, los recursos humanos y materiales; desarrolla la diversidad y la capacidad del entorno; $y$ tiene un compromiso activo con el entorno.

Un líder transformacional puede producir un alto nivel de motivación en los estudiantes, quienes pueden ser inspirados en la búsqueda de metas de largo plazo que habrán de contribuir a su exitosa inserción en la competitiva sociedad del conocimiento.

La Figura 1 ilustra las relaciones entre las demandas de la sociedad del conocimiento para los estudiantes y los roles del profesorado: 


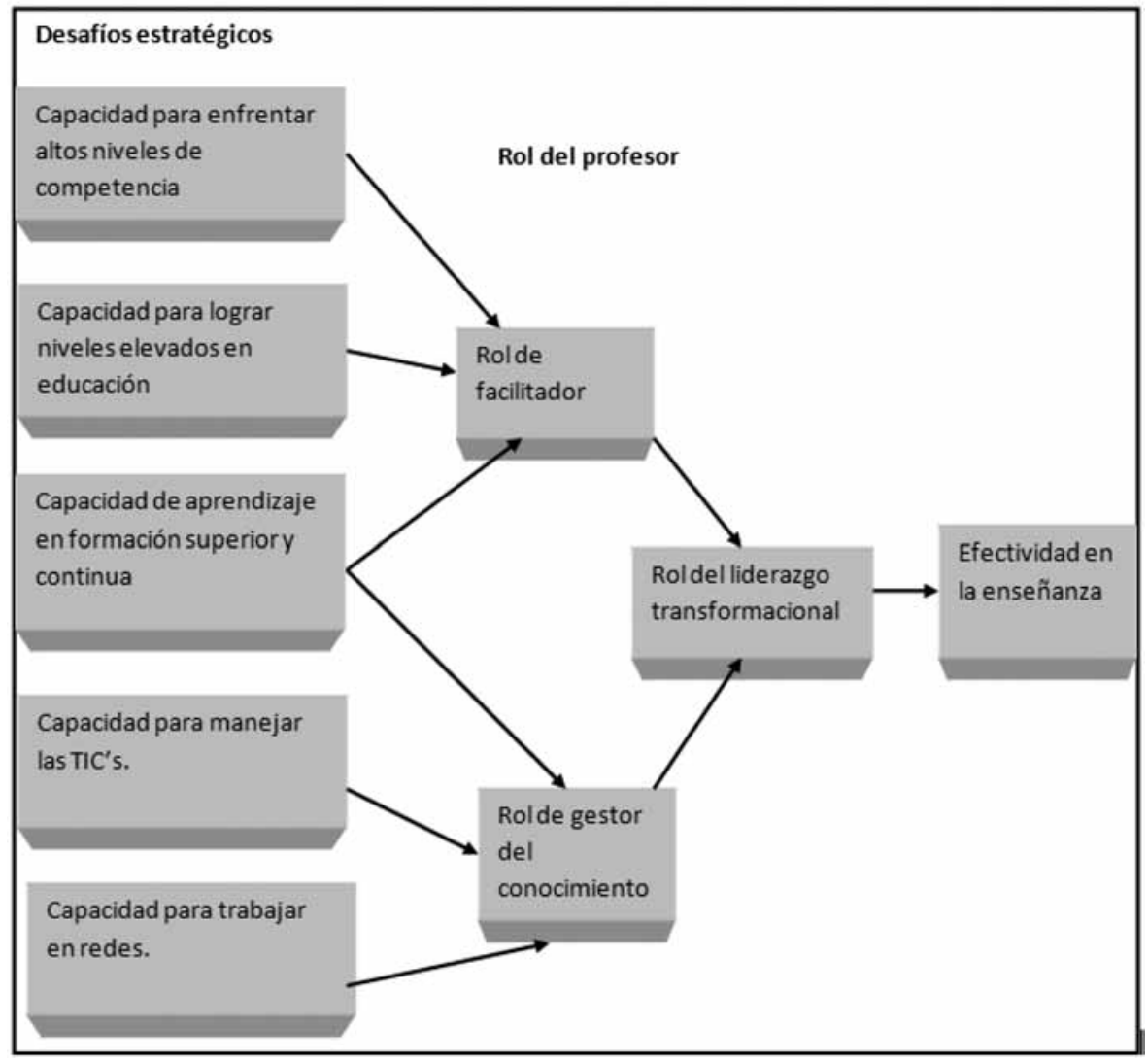

Figura 1. Relaciones entre las demandas de la sociedad del conocimiento para los estudiantes y los roles del profesorado.

Fuente: Elaboración propia.

\section{TENSIONES ASOCIADAS}

El desarrollo de una adecuada labor para los docentes del siglo XXI no está exento de tensiones. Como ha sido mencionado en los apartados anteriores, dejar atrás el paradigma de la sociedad del progreso industrial y pasar a la actual sociedad exige una multiplicidad de roles al docente.

Este desafío de asumir múltiples roles tales como facilitador, gestor de conocimiento, y líder transformacional, implica la existencia de una serie de tensiones.

En primer lugar, la satisfactoria preparación del profesorado puede ser un punto de quiebre. En efecto, en Chile al segundo año de funcionamiento de la ley de aseguramiento de la calidad, menos de un tercio de las carreras pedagógicas se encuentran acreditadas. En el año 2004 de 20 mil profesores que se formaban en el país, más de diez mil lo hacían bajo la modalidad de programas académicos especiales de corta duración y sin requisitos de ingresos más allá de la enseñanza media completa. No es de extrañar entonces que en los profesores jóvenes suele estar presente el sentimiento de cierto desamparo para realizar sus obligaciones [9].

En segundo lugar, es posible que los valores del profesor no sean plenamente coherentes con las exigencias de la sociedad del conocimiento y los roles que emergen de ellas. Por ejemplo, los profesores pueden optar por la filosofía de vida de no asumir la existencia de un mundo competitivo, pueden rechazar las implicancias de la globalización, y pueden incluso desistir de estimular a los estudiantes a entrar en una carrera vertiginosa de crecimiento y progreso de acuerdo a los cánones de la sociedad neoliberal. 
En tercer lugar, es posible que las capacidades intrínsecas del profesor no sean compatibles con los roles exigidos en esta propuesta. Por cierto, si bien las dotes de liderazgo pueden ser enseñadas y aprendidas, no es menos cierto que el líder transformacional tiene condiciones internas que emanan de la propia identidad del individuo. $\mathrm{La}$ facilitación y/o la gestión del conocimiento tampoco son labores triviales asumibles por cualquier persona; ambas demandan entrenamiento y capacidad personal.

En cuarto lugar, no siempre se cuenta con el apoyo de la alta dirección para realizar estas tareas. Así, el trabajo de Murillo [27] indica que el comportamiento y la actitud de la persona que asume las funciones de dirección en la escuela son un elemento fundamental que determina la existencia, la calidad y el éxito de procesos de cambio.

Los profesores que se encuentran en sus primeros años de ejercicio profesional viven a menudo esta realidad. Por ejemplo, Ávalos y Aylwin [3] al realizar un estudio cualitativo con nuevos profesores, mediante distintas técnicas de recolección de información, descubren la falta de apoyo hacia dichos profesores para comenzar su labor de enseñanza.

En este mismo sentido, Merrill [26] propone que los profesores tengan una suerte de mediación de entrada para así poder contar con un "empoderamiento" en el aula de clase y por consiguiente motivar a los estudiantes.

En quinto lugar, es posible que se produzca una tensión, entre directores y profesores, por las condiciones de trabajo y las reales posibilidades de desarrollo de la labor docente.

Este es un tema central, puesto que en diversos estudios se afirma que las limitaciones de tiempo y espacio en la escuela afectan negativamente los roles de liderazgo de los profesores (Mouer, 1999; Fay, 1992; Lieberman, et al., 1988; Wasley, 1989, referenciados en [7]). En este mismo sentido, Leech y Fulton [22] proponen la utilidad de crear al interior de las escuelas, a través de los directores, programas que muestren las experiencias que mejoren a los líderes potenciales. Con ello puede ser factible generar la habilidad de crear organizaciones de aprendizaje bajo el contexto de la sociedad del conocimiento.

\section{CONCLUSIONES}

A lo largo del trabajo se ha pretendido dar respuestas preliminares a las interrogantes definidas de la siguiente manera: ¿cuáles son los aspectos que caracterizan a la sociedad del conocimiento?; ¿cuáles son los principales roles que debe asumir un profesor para tender hacia la efectividad en la llamada sociedad del conocimiento?; ¿qué tensiones se producen al incorporar dichos roles?

Con el propósito de lograr el objetivo central consistente en identificar y describir las principales características de la sociedad del conocimiento que deben ser consideradas por el profesorado en el ejercicio de su actividad de enseñanza, se llevó a cabo un estudio de tipo conceptual en la literatura especializada.

A su vez, se logró la generación de un modelo preliminar que plasma relaciones entre las demandas de la sociedad del conocimiento para los estudiantes y los roles del profesorado. Dicho conjunto de relaciones se constituye en una base para la realización de un próximo estudio de tipo cualitativo, donde se pueda observar en la práctica de la labor docente si los aspectos descritos tienen la relevancia que el análisis conceptual ofrece.

Finalmente, como una potencial dirección de estudio futuro, se deja planteada la siguiente interrogante: ¿Cuáles serán las principales acciones estratégicas a implementar que permitan relevar el estatus del profesor en la sociedad del conocimiento?

\section{AGRADECIMIENTOS}

Este trabajo es el resultado del Proyecto FONDECYT 1090298, financiado por la Comisión de Investigación Científica y Tecnológica de Chile. La autora quiere agradecer la valiosa evaluación y sugerencias de la Dra. Beatrice Ávalos Davidson.

\section{REFERENCIAS}

[1] M. Alavi and D. Leidner. "Review: knowledge management and knowledge management systems: conceptual foundations and research issues". MIS quarterly. Vol. 25, Issue 1, pp. 107-136. 2001. 
[2] B. Ávalos. "La inserción profesional de los docentes". Revista de curriculum y formación de profesores. Vol. $13 \mathrm{~N}^{\mathrm{o}}$ 1, pp. 42-59. 2009. B. Ávalos and P. Aylwin. "How young teachers experience their professional work in Chile". Teaching and teacher education. Vol. 23, Issue 4, pp. 515-528. 2007.

[4] B. Bass and B. Avolio. "Improving organizational effectiveness through transformational leadership". Thousand Oaks, CA: Sage. 1994.

[5] A. Bassanini and S. Scarpetta. "Does human capital matter for growth in OECD countries? a pooled mean-group approach". Economics letters. Vol. 74, Issue 3, pp. 399-405. 2002.

[6] D. Béland. "Insecurity, citizenship, and globalization: the multiple faces of state protection”. Sociological theory. Vol. 23, Issue 1, pp. 25-41. 2005.

[7] V. Boyd-Dimock and K. Mcgree. "Leading change from the classroom: teachers as leaders". Issues about change, Vol. 4, Issue 4. Southwest Educational Development Laboratory. SEDL. 1995.

[8] J.J. Brunner, J. Bonnefoy, G. Elacqua and S. González. "Capital humano en la Región de Tarapacá". Universidad Adolfo Ibáñez. 2006.

[9] N. Can. "The leadership behaviours of teachers in primary schools in turkey". Education. Vol. 129, Issue 3, pp. 436-447. 2009.

[10] S. Dowrick and M. Rogers. "Classical and technological convergence: beyond the solow-swan growth model". Oxf. Econ. Pap. Vol. 54, Issue 3, pp. 369-385. 2002.

[11] P. Drucker. "La sociedad post capitalista". Colombia: Editorial Norma. 1994.

[12] S. Duryea, O. Jaramillo and C. Pages. "Latin American labor markets in the 1990s, deciphering the decade". IDB, Working Paper. 2003.

[13] J.M. Esteve. "Identidad y desafíos de la condición docente: vocación, trabajo y profesión en el siglo XXI”. En E. Tenti Fanfani (compilador). "El oficio de docente: vocación, trabajo y profesión en el siglo XXI". Buenos Aires, Argentina. osde-iipe/ unesco, pp. 19-69. 2006.

[14] B. Fainholc. "Teaching and learning in the knowledge society". Encounters on education. Vol. 6, pp. 87-105. 2005.
[15] D. Frantzen. "R\&D, human capital and international technology spillovers: a crosscountry analysis". Scandinavian Journal of Economics. Vol. 102, Issue 1, pp. 57-75. 2000.

[16] K. Hampton and B. Wellman. "Neighboring in netville: how the internet supports community and social capital in a wired suburb". City \& Community, Vol. 2, Issue 4, pp. 277-311. 2003.

[17] A. Hargreaves. "Teaching in the knowledge society: Education in the age of insecurity". Open University Press. New York, USA. 2003.

[18] A. Hargreaves and D. Fink. "El liderazgo sostenible, siete principios para el liderazgo en centros educativos innovadores". Ediciones Morata. España. 2008.

[19] A. Hargreaves and D. Fink. "The seven principles of sustainable leadership". Educational leadership. Vol. 61, Issue 7, pp. 8-13. 2004.

[20] W.C. Kim and R. Mauborgne. "Procedural justice, strategic decision making, and the knowledge economy". Strategic Management Journal. Vol. 19, Issue 4, pp. 323-338. 1998.

[21] E. Lamo de Espinosa. "Bajo puertas de fuego. El nuevo desorden internacional". Taurus. Madrid, España. 2004.

[22] D. Leech and R. Fulton. "Faculty perceptions of shared decision making and the principal's leadership behaviors in secondary schools in a large urban district". Education. Vol. 128, Issue 4, pp. 630-644. 2008.

[23] N.G. Mankiw, D. Romer and D. N. Weil. "A contribution to the empirics of economic growth". Quarterly Journal of Economics. Vol. 107, Issue 2, pp. 407-437. 1992.

[24] M.J. Marabotto. "Estrategias cognitivas y metacognitivas para las tecnologías de la información". Actas de las Jornadas de Informática Educativa 96, pp. 51-59. UNED. Madrid, España. 1996.

[25] C. Marcelo. "Aprender a enseñar para la sociedad del conocimiento". Revista Complutense de Educación. Vol. $12 \mathrm{~N}^{\mathrm{o}} 2$, pp. 531-593. 2001.

[26] M.L. Merrill. "No more sacrifices on the alar of educational excellence: ADR \& At-Risk Students. Ohio St. J. on disp. resol. 1993. 
[27] F.J. Murillo. "Una dirección escolar para el cambio: del liderazgo transformacional al liderazgo distribuido". Revista Iberoamericana sobre Calidad, Eficacia y Cambio en Educación. REICE, Vol. 4, No 4e, pp. 11-24. 2006. URL: http://www.rinace.net/arts/ vol4num4e/art2.pdf. Fecha de consulta: 25 de junio de 2009.

[28] J. Oliver. "Espacios educativos y sistemas de formación: metodología ecológica y organización educativa”. Revista Interuniversitaria de Formación del Profesorado. No 4, pp. 59-67. 1989.

[29] L. Pedraja-Rejas, E. Rodríguez-Ponce and M. Barreda. "Estilos de liderazgo y resultados del sistema de medición de la calidad de la educación: un estudio empírico en los colegios básicos de la ciudad de Arica-Chile". Ingeniare. Revista chilena de ingeniería. Vol. $17 \mathrm{~N}^{\circ}$ 1, pp. 21-26. 2009.

[30] L. Pedraja-Rejas, E. Rodríguez-Ponce and J. Rodríguez-Ponce. "Sociedad del conocimiento y dirección estratégica: una propuesta integradora". Interciencia. Vol. $31 \mathrm{~N}^{\circ} 8$, pp. 570-576. 2006.

[31] E. Rodríguez-Ponce. "El rol de las universidades en la sociedad del conocimiento y en la era de la globalización. Evidencia desde Chile". Interciencia. Vol. $34 \mathrm{~N}^{\circ} 11$, pp. 822-829. 2009.

[32] S. B. Sarason. "Teaching as performing art". New York: Teachers College Press. 1999.

[33] J.M. Tylee. "Nursing education in the tertiary sector in new South Wales, 1986-1989: an analysis of ideological orientations of curriculum, with particular reference to one institution". Unpublished Doctoral Thesis. The University of Newcastle. Australia. 1992.

[34] K. Uibu and E. Kikas. "The roles of a primary school teacher in the information society". Scandinavian Journal of Educational Research. Vol. 52, Issue 5, pp. 459-480. 2008.

[35] R. Van den Berg. "Teachers' meanings regarding educational practice". Review of Educational Research. Vol. 72, Issue 4, pp. 577-625. 2002.

[36] J. Villanueva. "La filosofía y la formación docente hacia la construcción y consolidación de una praxis educativa más consciente, crítica y participativa”. Laurus. Revista de Educación. Vol. 12 No extraordinario, pp. 206-235. 2006. 